\author{
P. H. WINKELMAN
}

\title{
RATIONALITÄT UND NORMATIVER GEGENDRUCK IM WIRTSCHAFTLICHEN INTERNATIONALISMUS $1850-195^{\circ}$
}

Gegen Mitte des neunzehnten Jahrhunderts war das wirtschaftliche Denken so weit fortgeschritten, daß es ein theoretisches System bildete, wobei die Regeln, die beim internationalen Güteraustausch zu beachten sind, formuliert wurden, zur Verwirklichung dessen, was als die natürliche Ordnung des Außenhandels angesehen wurde. Drei Probleme oder Gruppen von Problemen waren bis dahin untersucht worden.

I.) Welche sind die Triebfedern des zwischenstaatlichen wirtschaftlichen Verkehrs? Privathändler importieren und exportieren, weil sie im einen Lande billig einkaufen können und durch Verkauf in einem anderen Lande Gewinn erzielen. Es stellte sich die Frage, wie es komme, daß die Preise dieser Güter im einen Lande niedriger liegen als im anderen.

2.) Das zweite Problem drehte sich um die Finanzierung des Außenhandels. Jeder Produzent verlangt für die Güter, die er verkauft, Zahlung in der Währung des eigenen Landes. Der Importeur im Ausland bezahlt in der Währung seines Landes. Die Frage, um die es sich handelt, ist nun, wie die Umsetzung einer Währung in die andere stattfindet. Der Privathändler macht sich aus diesem Prozeß kein Problem. Sogar die Bankiers, die den Geldübermittlungsdienst durchführen, interessiert es nicht, welchen Einfluß ihre Transaktionen auf die eigene Währung oder auf die Preise von Gütern und Dienstleistungen, ausgedrückt in der eigenen Währung, ausüben. Schließlich interessiert sie ebensowenig der Einfluß auf die wirtschaftliche Aktivität und die Arbeitsgelegenheit im eigenen Lande.

3.) Die dritte Gruppe von Problemen bezieht sich auf die Verteilung der Vorteile, die den verschiedenen Ländern aus dem Außenhandel zufließen. Auch diese Frage ist für die Händler und die Bankiers kein Problem. Sowohl der Import als auch der Export bringen Gewinn. Welchen Einfluß jedoch hat ein solcher Handel auf das Wohlstands- 
niveau der Mitbürger und auf die nationale Gemeinschaft als Ganzes? Der Import tritt, direkt oder indirekt, mit den Erzeugnissen der eigenen Ökonomie in Wettbewerb. Steht sich die nationale Gemeinschaft besser, wenn die Importe billiger sind als die eigenen Erzeugnisse, oder etwa schlechter, wenn der Preis der einheimischen Produkte gesenkt wird?

Die Antwort auf die erste Frage wurde in dem Lehrsatz von den komparativen Kosten gefunden, in dem das Limit nach oben und unten bestimmt wurde, dergestalt, daß den Geschäftspartnern aus ihrem gegenseitigen Handel Vorteil erwächst. Stuart Mill fügte noch hinzu, daß auch im zwischenstaatlichen Handelsverkehr das Gesetz von Angebot und Nachfrage gelte. Innerhalb der durch die Produktionskosten bestimmten Grenzen werde sich der Preis auf ein Niveau stellen, das durch die Intensität der Nachfrage in beiden Ländern bedingt sei.

Das Problem der Finanzierung des internationalen Handelsverkehrs wurde mit einer finanziellen Theorie beantwortet, die ursprünglich auf David Hume zurückgeht. Die nationalen Geldsysteme sowie die Preise sind durch die Zahlungsbilanz miteinander verbunden. Der Austausch von Edelmetallen bewirkt die Anpassung der Preise und bestimmt den Umfang des Handels. Die Bewegung der Edelmetalle reagiert automatisch auf die Bedürfnisse des Handels. Wenn in einem bestimmten Land die Preise steigen, wird zum Ankauf von Waren im Ausland Edelmetall gebraucht. Die Folge der Ausfuhr von Edelmetallen ist, daß die Preise sinken. Zwischen der Gold- und Güterbewegung besteht also eine Wechselwirkung, welche die Aufrechterhaltung des Gleichgewichts in der Zahlungsbilanz gewährleistet.

Nassau Senior erläuterte diesen Satz mit einem Beispiel. England hat 5.000.000 Pfund Sterling nach Frankreich exportiert zum Ausgleich einer Importrechnung. Was wäre die Folge davon? Gesetzt den Fall, dieses Gold würde nicht in den französischen Geldumlauf aufgenommen und keine monetäre Expansion zur Folge haben. Welchen Nutzen hätte Frankreich davon? Es gibt nur zwei Möglichkeiten. Wird das Gold wohl in die umlaufende Geldmenge aufgenommen, so verursacht es einen Anstieg der Preise und eine Erhöhung der Einfuhren in Frankreich. Wird es nicht in den Geldumlauf aufgenommen, so hätte Frankreich für seine Ausfuhren ein nutzloses Gut empfangen. Einlagerung und Aufbewahrung bringen nur Kosten mit sich. Dagegen hätte England für 5.000.000 Pfund Sterling an Gütern empfangen, die für den Konsum zur Verfügung stehen. Senior glaubte nicht, daß je ein Volk so töricht sein würde, wertvolle Produkte gegen Gold einzutauschen und dann dieses Gold in teuren Kellern zu vergraben. 
The tail is wagging the dog. Der Hund läuft hinter dem Schwanz. ${ }^{1}$

Das dritte Problem bezog sich auf "the gains from trade", die Verteilung der Vorteile unter die handeltreibenden Länder. Die Antwort war in der Theorie von der gegenseitigen Nachfrage enthalten. Jedes Land sollte denjenigen Teil bekommen, der dem Konkurrenzvermögen seiner Industrien entsprach. Diese Antwort genügte ohne weiteres. Den Fragen der nationalen Vorteile wurde nur geringe Aufmerksamkeit gewidmet. Das Interesse galt einer möglichst großen Expansion des Handels weit mehr als den Berechnungen, wie diese Vorteile nun eigentlich genau zu verteilen wären. Wichtig war nur, daß die produktiven Kräfte der Welt so gut wie möglich ausgenutzt werden sollten. $\mathrm{Zu}$ diesem Zwecke bedurfte es des Welthandels. Universeller Freihandel würde allen Ländern Wohlstand bringen. Man hatte keine Angst vor Importen, keine Furcht, daß Goldverlust zu einer ungünstigen Zahlungsbilanz führen möchte, Preissenkungen und Arbeitslosigkeit hervorrufen würde. Zum Teil erklärte sich diese Einstellung aus der Orientierung auf ein Gleichgewicht auf lange Sicht, aber hauptsächlich beruhte das Vertrauen auf den heilsamen Kräften der Konkurrenz. ${ }^{2}$

Die Rationalität dieses Komplexes von Lehrsätzen hat zwei Aspekte. Sie beruht auf einer wirtschaftlichen Analyse nach der Methode der isolierenden Abstraktion. Diesen Abstraktionen lag die philosophische Überzeugung zugrunde, daß es eine natürliche Ordnung der Dinge gebe, die sich auf die Vernunft stütze. Die Kritik an der Logik der wirtschaftlichen Gedankengänge hat sich immer auf die Zulässigkeit der Annahmen gerichtet, deren Anwendbarkeit von dem System abhängig war. Also eine Beurteilung der Rationalität im Sinne von zweckmäßig, vorteilhaft. Eine zweite Bedeutung von Rationalität ist Billigkeit, worin eine normative Regel, Regeln von Rechtmäßigkeit und Sichgehören beschlossen liegen.

Die Frage nach der Zweckmäßigkeit fällt hier nur insofern ins Gewicht, als sie die Billigkeit begrenzt oder ein sie mitbestimmendes Element bildet. Es leuchtet ein, daß die monetäre Analyse nur dann Wirklichkeitswert besitzt, wenn es ein Geldsystem gibt, bei dem Geld und Gold gegenseitig ersetzbar sind. Die Ausdrücke ,money" und „precious metals" wurden durcheinander verwendet. Die englische Coinageakte des Jahres I 816 war mit dieser Identifikation weitgehend

1 Nassau Senior, Three lectures on the transmission of the Precious Metals from Country to Country, I828; vgl. J. B. Condliffe The Commerce of Nations, London, I95 I, S. I93. 2 Gerhard Stavenhagen, Geschichte der Wirtschaftstheorie, Göttingen, 1957, S. 40I-4I I; J. B. Condliffe, a.a.O., S. I69-202; Joseph Schumpeter, History of Economic Analysis, London, 1955, S. 373,;605-616; J. F. Bell, A History of Economic Thought, New York, 1953, S. $182-183,236-237,269-270$. 
in Übereinstimmung. Das Pfund Sterling wurde an das Gold gekoppelt, aber das Gold seinerseits an das Pfund Sterling. Damit wurde eine Weltvaluta geschaffen, die dem kosmopolitischen Ideal der Theorie entsprach.

Weniger gut mit der Wirklichkeit vertrug sich die Lehre von den komparativen Kosten. Sie beruhte auf einer Anzahl Suppositionen, von denen gerade den zwei wichtigsten eine gewisse Verschrobenheit anhaftete. Die erste war, daß Kapital und Arbeit innerhalb der nationalen Grenzen mobil seien und sich zwischen verschiedenen Ländern gar nicht bewegten. Weder das eine noch das andere entsprach zur Genüge der Wirklichkeit. Wohl aber bestand im internationalen Verkehr eine größere Immobilität der Produktionsfaktoren als innerhalb der nationalen Grenzen. Diese relative Immobilität genügte, um dennoch einen komparativen Vorteil entstehen zu lassen. Demgegenüber stand wieder, daß Kapital mobiler ist als Arbeit. Das hat zur Folge, daß der Handel mit dem Ausland die Verteilung des Nationaleinkommens zum Nachteil des Arbeitseinkommens beeinflußt. Neue Arbeitsmöglichkeit für arbeitslos gewordene Arbeiter ist, namentlich auf kurze Sicht, nicht immer gewährleistet. Hier verstrickte sich das abstrakte Optimum an Zweckmäßigkeit in die konkreten Ansprüche der Billigkeit.

Die zweite wichtigste Supposition, welche der Lehre von den komparativen Kosten zugrunde lag, war die Lehre vom Arbeitswert. Produktionskosten sind Arbeitskosten. Zu Adam Smiths Lebzeiten konnte die Maschine noch als ein Fortsatz des Menschen betrachtet werden, aber ein halbes Jahrhundert später wurden die Rollen allmählich vertauscht. ${ }^{1}$ Der Wirklichkeitswert konnte damals nicht mehr hoch angeschlagen werden. Ricardo hat versucht, die Schwierigkeit zu verkleinern, indem er das Kapital als das Produkt voraufgehender Arbeit betrachtete und die Kosten auf Arbeitskosten zurückführte. ${ }^{2}$ Stuart Mill erreichte sehr viel mehr. Er behandelte den Güteraustausch als Ausgleich der internationalen Nachfrage; damit geriet die Lehre vom Arbeitswert völlig in den Hintergrund. ${ }^{3}$ In dieser Verbesserung hat sich das Theorem von den komparativen Kosten, nachdem man die Lehre vom Arbeitswert längst aufgegeben hatte, behaupten können.

In dem dritten Lehrsatz vom Außenhandel, dem von der Verteilung der Vorteile auf die Handelspartner, verlagert sich der Akzent in der Rationalität von der Zweckmäßigkeit nach der Zulässigkeit und der Rechtmäßigkeit. Die Auffassung von „the gains from trade” ist nicht in derselben Bedeutung ein Lehrsatz wie die monetären Betrachtungen

1 L. J. Zimmerman, Geschiedenis van het economisch denken, 5. Aufl, Haag, 1957, S.276.

Staatslexicon, Recht, Wirtschaft, Gesellschaft, Bd. IV, Freiburg, x959, S. 397.

${ }^{3}$ G. Stavenhagen, a.a.O., S. 408. 
über die automatische Anpassung der Preise und wie der Lehrsatz von den komparativen Kosten und der gegenseitigen Nachfrage. Eigentlich war sie eine aus diesen sich ergebende Schlußfolgerung, allerdings eine Schlußfolgerung mit selbständiger Bedeutung. Schon in dem lebhaften Interesse für den rein wirtschaftlichen Nutzeffekt, den man sich vom Auslandshandel versprach, näml. die Aktivierung ungenutzter Produktionskräfte in der ganzen Welt, lag ein humanitäres Normbewußtsein, das mit der Theorie und der Geschichte der voraufgehenden Jahrhunderte kontrastiert.

Aber auch der intellektuelle und moralische Wert des Wirtschaftsverkehrs stand damals hoch im Kurs. Wie früher der Krieg, so ist heute der Handel der wichtigste Anlaß zum Kontakt zwischen den Völkern, schreibt Stuart Mill. Der Handel hat die Menschheit gelehrt, den Reichtum und den Wohlstand anderer Länder mit Wohlwollen anzusehen. Früher wünschte sich der Patriot, daß alle Länder schwach, arm und schlecht regiert wären, nur nicht das seine. Heute sieht er in deren Reichtum eine unmittelbare Quelle des Fortschritts für das eigene Land. Der Handel nimmt dem Krieg seine wichtigsten Triebfedern. Er festigt und vervielfältigt die persönlichen Interessen, die den Krieg nicht wollen. Die schnelle Ausdehnung des internationalen Handels ist die wichtigste Gewähr für den Weltfrieden, den Fortschritt und die Zivilisation. ${ }^{1}$

Das schwache Interesse an dem Verteilungsproblem hat dieselbe Ursache wie die national-ökonomischen Lehrsätze von der Verteilung der Gewinne und der Höhe der Löhne. Dieser Prozeß verläuft gleichfalls gesetzmäßig, weil sämtliche wirtschaftlichen Wahlhandlungen von nur ein und demselben Prinzip beherrscht werden: aus den gegebenen Möglichkeiten einen möglichst großen Profit zu ziehen. Nun hat aber eben dieses Prinzip die herrschende Richtung in der Ökonomie am meisten diskreditiert. Der optimistische Fortschrittsglaube war kein allgemeiner. So war z.B. Schopenhauer der Ansicht, daß es eine schlechtere Welt als die bestehende nicht geben könne. ${ }^{2}$ Es wäre verwunderlich, wenn dieselben Prinzipien international-ökonomisch das Idyll eines Hand-in-Hand-Gehens von Zweckmäßigkeit und Billigkeit geschaffen hätten, dagegen aber national-ökonomisch die Ursache des Versagens der Ökonomie als sozialer Wissenschaft gewesen wären.

Zum guten Verständnis dieses angenommenen Paradoxons muß man unterscheiden zwischen dem homo economicus, wie ihn die Legende überliefert hat, und wie er in Wirklichkeit gewesen ist. Zum

1 John Stuart Mill, Principles of Political Economy, vgl. J. B. Condliffe, a.a.O., S. I98.

'R. B. Bailey, Sociology faces pessimism, Haag, I958, S. 48. 
Teil verdankt er seine legendäre Gestalt den Ausdrucksweisen der klassischen Autoren. Stuart Mill war der erste, der ausdrücklich feststellte, daß die Ökonomie sich nicht mit dem ganzen Menschen befaßt, sondern nur mit dem Menschen, insofern er nach Reichtum strebt. Die Ökonomie basiert auf einer willkürlichen Definition vom Menschen als einem Wesen, das unveränderlich dasjenige tut, was ihm mit dem geringsten Aufwand an Arbeit und physischer Anstrengung das größte Quantum lebensnotwendiger Güter und Luxusartikel einbringt, die er bei dem jeweiligen Stand der Kenntnisse erwerben kann. ${ }^{1}$

Es versteht sich, daß man auf Grund einer solchen Begriffsbestimmung leicht dazu kam, das ökonomische Subjekt für eine Art Roboter zu halten, ohne Herz und ohne Gewissen. In seinen Augen gibt es kein Unrecht, er fürchtet nur die Justiz und um des Geldes willen vernachlässigt er seine Gesundheit. Konsumtion und Etholung wären lediglich Mittel zum Gelderwerb. Nie würde er zur Befriedigung nicht-essentieller Bedürfnisse oder für wohltätige und andere Zwecke in großzügiger Weise Geld ausgeben. Er würde jeden Augenblick seines Lebens dermaßen ausnützen, daß er am Ende seines Lebens einen größtmöglichen Reichtum besitzen würde.

Die Analyse der Werke klassischer Autoren hat gezeigt, daß der so hingestellte homo economicus eine Karikatur ist. Allwissenheit und eiserne Willenskraft, erste Erfordernisse, um überall und immer den Maximalprofit zu sehen und zu wollen, hat ihm niemand je zugeschrieben. Schon der bloße Unterschied zwischen Marktpreis und natürlichem Preis, worüber die klassischen Autoren schreiben, beweist, daß sie das Wirtschaftsleben nicht mit den Abstraktionen der Theorie identifizierten.

Ihre Meinung geht vielmehr dahin, daß die Ökonomie eine Theorie ist für das Verhalten des Menschen bei seinen wirtschaftlichen Entscheidungen, immerhin mit der Einschränkung, daß er nicht um jeden Preis nach einem größtmöglichen Vermögen strebt, sondern nach einem größtmöglichen Einkommen aus einer gegebenen Anzahl Hilfsmitteln. Die Theorie abstrahiert nicht von altruistischer Anwendung eines einmal erworbenen Geldeinkommens, wohl aber von altruistischen Neigungen, die mit der Erwerbung eines größtmöglichen Einkommens in Konflikt geraten, z.B. von dem Altruismus, der sich auf den Tauschpartner richtet. Der homo economicus lebt nur als in der isolierenden Abstraktion gebildete Idee und hat mit der generalisierenden Abstraktion der psychologischen Wissenschaft nichts zu schaffen. Letztere sieht ihn als einen Menschen von bestimmtem

1 P. Hennipman, Economisch motief en Economisch principe, Amsterdam, 1945, S. 42-59. 
Charaktertyp, der sich durch einen Komplex von Neigungen und Eigenschaften, welche in seinem ganzen Leben zutage treten, kennzeichnet.

Durch diese Korrektionen ist die Anwendbarkeit des Modells nicht unerheblich größer geworden. Der Typ entspricht dem Bild des Durchschnittskaufmanns aller Zeiten. Dieser Durchschnittskaufmann agiert auf der internationalen Schaubühne genauso wie vor seinen Tauschpartnern im eigenen Lande. Sein ganzes Auftreten wird von dem gängigen wirtschaftlichen Prinzip des alternativen, rationellen Wählens zwischen den verschiedenen Anwendungsmöglichkeiten knapper Produktionsmittel bestimmt. Bis soweit wird er der Billigkeit immer noch gerecht. Der Tausch kommt nur dann zustande, wenn beide Partner in ihm Vorteil sehen. Handelskontakt ist ein vollwertiger, menschlicher Kontakt. Aus Geschäftsverbindungen entstehen Geschäftsfreunde, oder die Verbindungen werden abgebrochen.

Es verbleibt nunmehr die Frage, worin nach dem Maßstab der Gerechtigkeit der Unterschied zwischen nationaler Ökonomie und internationalem Verkehr bestehe. Darüber kann uns die Geschichte näher aufklären. Der homo economicus des angehenden neunzehnten Jahrhunderts war der Zeitgenosse des Rechtsstaats, der aus derselben Welt des Denkens stammt. Der Rechtsstaat schuf die Voraussetzungen für die ökonomischen Subjekte, dem wirtschaftlichen System entsprechend zu handeln, und zwar intern: die Freiheit des Berufs, die freie Konkurrenz und den Schutz des Eigentums. Extern wurden diese Rechte in passender Weise erweitert mit einem liberalen Fremdenrecht und einer pünktlichen Befolgung der Regeln der internationalen finanziellen Technik. ${ }^{1}$

So, wie der externe Güteraustausch unter diesen Bedingungen verlief, bestand mit dem System der voraufgehenden Jahrhunderte ein himmelweiter Unterschied. Die Tauschpartner hatten sich damals auch von wirtschaftlichen Erwägungen leiten lassen, allein ihre Bewegungsfreiheit war durch Staatsintervention reguliert. Auch intern gab es politische Einmischung, aber wesentlich ist, daß interne und externe Regulierung zusammen der Nationalökonomie vor allem Ausländischen den Vorrang verlieh. ${ }^{2}$ In dem neuen System wurden die Rollen vertauscht. Wenn der internationalen Arbeitsteilung und der Nivellierung der Produktionsfunktionen Tür und Tor geöffnet werden, so bedeutet dies, daß der internationale Verkehr den Primat über die nationale Wirtschaft erlangt. Dieses Verhältnis ist für den Wert des Systems

1 Georg Erler, Grundprobleme des internationalen Wirtschaftsrechts, Göttingen, I956, S. $43-70$.

${ }^{2}$ Edmund Silberner, La Guerre et la Paix dans l'histoire des Doctrines Economiques. Paris, 1957, S. XXVII-XXXVII. 
von entscheidender Bedeutung. Das System hat in allen Teilen der Welt die Produktionsfaktoren wie niemals zuvor aktiviert. Die Kehrseite war, daß dem Nationalstaat zur Erfüllung seiner Recht schaffenden Funktion zu wenig Spielraum übrigblieb. Diese Grenze war der Rationalität, in der Bedeutung von Billigkeit, bis zum Ende der Periode gesetzt.

Jérome Adolphe Blanqui, der Say am Collège de France nachfolgte, prophezeite im Jahre I 838, daß die Terminologie und die analytischen Methoden der englischen Autoren überall als die Sprache, deren sich die theoretische OOkonomie bedienen sollte, anerkannt und angenommen werden würden. In der italienischen und französischen Schule stellte er eine Vermischung von wirtschaftlichen mit philosophischen Elementen fest. Dort sprach man über Ökonomie in ihrer Beziehung zum Gemeinwohl, über die gesellschaftlichen Reformen und über die ewigen Prinzipien von Gerechtigkeit bei der Verteilung der Früchte der Arbeit.

Obige Prophezeiung hat sich erfüllt, aber die exakte Definition wirtschaftlicher Begriffe hat keineswegs dazu geführt, daß überall in der Welt aus der isolierenden Abstraktion des wirtschaftlichen Motivs und des wirtschaftlichen Prinzips dieselben Schlüsse gezogen wurden. Alexander Hamilton formulierte i79I das Erziehungsmotiv zum Schutz der Industrien in weniger entwickelten Ländern. Eine Generation später war Mathew Carey eifrigst bestrebt, diesem Prinzip in der Handelspolitik Eingang zu verschaffen. In Deutschland wurde das Motiv an den politischen Nationalismus gekoppelt. Eine dritte Gruppe von Kritikern bildeten die kolonialen Reformer. ${ }^{1}$ All diese Autoren kritisierten jedoch im Rahmen der bestehenden wirtschaftlichen Ordnung. Die Zweckmäßigkeit wurde zwar nicht bestritten, aber in Anbetracht der Umstände abgelehnt. Die Prüfung der Billigkeit war nur möglich, wenn der Internationalismus als eine Fortsetzung des Gegensatzes zwischen Lohn und Gewinn betrachtet wurde. Die Billigkeitskritik ist bei den Sozialisten entstanden.

Diese Linie nimmt bei Johann Gottlieb Fichte ihren Anfang. In seinem Buch „Der geschlossene Handelsstaat”, Tübingen 1800 , finden sich bereits wesentliche Elemente des späteren sozialistischen Denkens. Innerlich war er ein Anhänger der persönlichen, freien Entfaltung im wirtschaftlichen Handeln. Insoweit kämpft er mit vielen anderen Zeitgenossen gegen die sinnlos gewordenen Regulierungen des Mer-

1 J. B. Condliffe, a.a.O., S. 237-259. 
kantilismus und gegen die organisierten Interessengruppen, welche die Gewinne eingestrichen hatten. Aber nicht weniger nachdrücklich betonte er die Gleichheit. Sein Buch erschien drei Jahre nach dem Prozeß gegen Gracchus Baboeuf, von dem er wahrscheinlich vieles übernommen hat. Fichte glaubt an die Möglichkeit eines Systems natürlicher Preise, wobei einerseits die Produzenten für ihre Güter und Dienstleistungen völlig entschädigt werden, andererseits eine billige und gleichmäßige Verteilung des Nationaleinkommens garantiert wird. Das erfordert Überwachung der Inlandspreise durch den Staat. Hieraus folgt, daß Preisschwankungen und Abweichungen von dem wirklichen Wert der Güter hauptsächlich aus der Wechselwirkung $z w i s c h e n$ der Nationalökonomie und der Außenwelt erwachsen werden. Internationaler Handel macht eine zielgerichtete Regelung der Produktion, der Preise und der Distribution unmöglich. Die einzige Abhilfe kann nur totale Isolierung vom Ausland schaffen. Bei Fichte findet sich zum erstenmal der Gedanke, daß der Außenhandel ein Staatsmonopol sein soll.

Bei Fichte sucht man noch vergebens nach einem Abwägen der Vorteile der internationalen Arbeitsteilung und der Opfer, die gebracht werden müssen, um der Billigkeit gerecht zu werden. Bei Marx findet man dieses Abwägen wohl. Er hat sich dahin geäußert in einem Vortrag vor dem "Demokratischen Club" in Brüssel im Jahre I 848. Marx kannte den Lehrsatz von den komparativen Kosten, aber seiner Ansicht nach war die Kostenersparnis nicht heilsam, wenn sie mit einem entsprechenden oder noch stärkeren Absinken der Löhne verbunden war. Gerade dies erwartete er von dem Sinken des Brotpreises. Es ist für den Arbeiter nicht gleichgültig, ob er nur vier Francs erhält, weil das Brot billiger geworden ist, wenn er zuvor fünf Francs bekommen hat. Die Löhne sind immer niedriger geworden im Vergleich zu den Gewinnen. Solange der Getreidepreis höher wat, brauchte der Arbeiter von seinem Brotkonsum nur wenig abzusparen, um sich andere Sachen anschaffen zu können. Sobald jedoch das Brot billig ist, und dadurch die Löhne niedrig sind, kann er vom Brot fast nichts absparen, um andere Artikel zu kaufen.

Der Fall des Brotpreises wird sogar eine mehr als verhältnismäßige Preissenkung der Geldlöhne zur Folge haben. Die Verstärkung produktiver Kräfte wird zur Konzentration des Kapitals und zu intensiverer Ausnützung der maschinalen Technik führen. Geschulte Arbeit wird verhältnismäßig weniger nützlich und die Konkurrenz in der ungeschulten Arbeit wird größer.

Marx zieht hieraus jedoch nicht den Schluß, daß der Freihandel bekämpft werden soll. Das geht aus seiner Auffassung von dem Prozeß der kapitalistischen Entwicklung auf lange Sicht deutlich hervor. Es 
gibt eine historische Parallele zwischen den schüchternen Anfängen des Kapitalismus und seiner Entwicklung zu der alles beherrschenden Form sozialer Organisation und daneben dem gleichzeitigen Wachsen des industriellen Proletariats. Die merkantilistische Protektion war notwendig gewesen als ein Mittel für die Fabrikanten, den unabhängigen Handwerkern ihren Besitz zu nehmen. Jetzt waren die Verhältnisse andere geworden. Der Freihandel hebt die Beschränkungen auf, welche die kapitalistische Entwicklung innerhalb der nationalen Grenzen beschlossen hält. Der Ausbeutung der Arbeiter durch die Kapitalisten macht er jedoch kein Ende. Die immer härtere Ausbeutung der Arbeiter in allen Ländern wird die internationale Vereinigung aller Proletarier zustande bringen, die der Anfang der sozialen Revolution sein wird. Im allgemeinen gesprochen, sagt er wörtlich, ist die Protektion in der heutigen Zeit beharrend, während das Freihandelssystem eine zersetzende Wirkung hat. Die Gegensätze zwischen dem Proletariat und der Bourgeoisie werden dadurch auf die Spitze getrieben. Nur in diesem revolutionären Sinne sei er ein Verfechter des Freihandels. ${ }^{1}$

Marx' Kritik richtete sich also nicht gegen die Logik der liberalen Analyse der internationalen Beziehungen. Bei den Anhängern des Protektionismus war dies ebensowenig der Fall. Sie bekämpften ihre Anwendbarkeit in der Welt wie sie nun einmal ist, eine Vielheit von Völkern und Kontinenten, gezwungen, im Wetteifer zu leben. Ihnen sagte der Primat der kosmopolitischen Ordnung nicht zu, weil er an Utopismus streifte. Marx gab bei dieser Kontroverse den wenig interessierten Zuschauer $a b$. Auch er ging von vorgefaßten Ideen aus; es waren jedoch nicht Ideen über die Notwendigkeit einer nationalen Gliederung der wirtschaftlichen Organisation, sondern über eine ideale, sozusagen apolitische, Gesellschaft. Diese Ideen hat er übrigens nicht näher ausgearbeitet. ${ }^{2}$ Ebenso wie die Nationalisten anerkannte er die theoretische Zweckmäßigkeit des Außenhandels und kritisierte er die dadurch hervorgerufene Unbilligkeit, eine Unbilligkeit nicht für die Nationalwirtschaft, sondern für die Arbeiterklasse. Wegen dieses abweichenden Motivs nahm dieses Problem in seinem Denken auch keine Zentralstellung ein. Wenn dereinst die ideale Gesellschaft begründet würde, würde die Streitfrage zwischen Freihandel und Protektion erledigt sein.

Bei dieser Überzeugung hat er beharrt. Die bestehende Gesellschaft ist ungerecht und angesichts dieses Ubelstandes macht es wenig aus, ob der nationale Staat mehr oder minder autonom seine Handelspolitik

1 Bert F. Hoselitz, Socialism, Communism and International Trade, The journal of political economy, Vol. 57 (I949) S. 227-232.

2 L. J. Zimmerman, a.a.O., S. I 12. 
bestimmt oder aber in Befolgung der Regeln des zwischenstaatlichen Verkehrs. Nur wenn das Problem die Aufmerksamkeit von dem großen Ziel ablenken sollte, erhebt er Protest. Im Jahre I 875 übte er Kritik an dem Programm von Gotha. Die deutschen Sozialisten nahmen bei dieser Gelegenheit als Aktionsprogramm an, daß die arbeitende Klasse ihre Befreiung in erster Linie im Rahmen des bestehenden nationalen Staates zu erlangen versuchen sollte. Das natürliche Ergebnis dieser Aktion würde „die internationale Brüderlichkeit aller Nationen" sein.

Natürlich, so sagt Marx, sollen sich die Arbeiter im eigenen Lande organisieren, um den Kampf führen zu können. Aber dies bezieht sich nur auf die Art und Weise des Kampfes, es bestimmt nicht dessen Inhalt. Es ist irreführend, von einer Aktion im Rahmen des bestehenden nationalen Staates zu sprechen. Der Staat selber muß handeln im Rahmen der internationalen wirtschaftlichen und politischen Verhälnissse. Wenn die Sozialisten die Losung „Brüderlichkeit der Nationen” in den Mund nehmen, so ist das nichts anderes als das Jargon, dessen sich der bürgerliche Pazifismus bedient. Die internationale Brüderlichkeit, welche die Sozialisten vor Augen halten sollen, ist die der Arbeiter aller Länder, nicht die der innerlich durch Klassenkampf geteilten Nationen. ${ }^{1}$

Die verschiedenen Auffassungen über den Sinn des wirtschaftlichen Internationalismus hängen mit den variierenden Ideen über Krieg und Frieden klar zusammen. Was Stuart Mill davon hielt, findet man bei allen Liberalen wieder. Sie glauben, daß der Krieg nicht durch unüberbrückbare nationale Gegensätze, sondern vielmehr durch schlecht verstandene nationale Motive verursacht wird. Der Krieg ist nur für diejenigen denkbar, die die Grundlagen der wirtschaftlichen Entwicklung nicht kennen. Nur ein ganz kleiner Teil der Bevölkerung zieht Nutzen aus einem Krieg. Es sind die kleinen Minderheiten, die aus Eigennutz den Krieg heraufbeschwören.

Die Wirtschaftswissenschaft dagegen ist die Wissenschaft vom Frieden schlechthin. Die Verbreitung wirtschaftlicher Kenntnisse hat die Tendenz, dem Krieg zu steuern. Dies soll nicht besagen, daß ein aufgeklärter Nationalismus bekämpft werden müßte, oder daß das Prinzip der Nationalitäten abgelehnt werden dürfte.

Über die tatsächliche Wirkung der liberalen Theorie auf das Kriegsproblem bestehen zweierlei Meinungen. Die größten Optimisten erwarten, daß alle Kriege oder so gut wie alle Kriege vermieden werden können. Es ist dies die Auffassung der Say, Dunoyer, Cobden und Bastiat. Andere dagegen wollen nicht weiter gehen, als daß sie die 1 G. D. H. Cole, Socialist Thought, Marxism and Anarchism, 1850-1890, London, 1957, S. 248-249. 
Gefahr eines drohenden Krieges für nicht mehr so groß halten: Ricardo, James Mill, Mac Culloch, John Stuart Mill, Chevalier, Baudrillart und Molinari. Nur Malthus anerkennt zwischen Freiheit und Frieden keinen Zusammenhang. Jedenfalls ist die Freiheit, die zu Reichtum führt, eine bessere Vorbereitung zum Kampf als die Protektion, die verarmt.

So wenig der wirtschaftliche Internationalismus einer internationalen Organisation bedarf, ebensowenig ist es nötig, daß politische internationale oder übernationale Organe gebildet werden, die mit einer zwingenden Autorität versehen werden. Gustave de Molinari ist der einzige, der eine solche Organisation für notwendig hält. Sie wird die Aufgabe haben, einen etwaigen Angreifer mit vereinten Kräften zu unterwerfen.

Nach Ansicht der Protektionisten ist der Krieg eine zwangsläufige Erscheinung in den Beziehungen zwischen den Nationen zur Erzielung reeller Vorteile. Mit Rücksicht auf die Vorteile des Krieges, halten sie für nicht wahrscheinlich, daß bewaffnete Konflikte aufhören werden. Nur ganz wenige halten die Möglichkeit für eine entfernte $\mathrm{Zu}-$ kunft offen. Vorläufig ist für die Unabhängigkeit, die Größe oder den Verfall der Nation ihre Wehrmacht entscheidend. Abrüstung ist unter solchen Umständen eine Torheit. Der Friede wird dadurch nicht gewährleistet. Die anderen werden nur zum Angriff ermutigt.

Die Sozialisten verwenden Ausdrücke, die an die der Liberalen erinnern. Krieg ist körperliches und geistiges Leiden und materieller Schaden. Solche Äußerungen berühren sich nahe mit der liberalen Kritik an dem wirtschaftlichen und politischen Nationalismus. Der Unterschied liegt in den Ursachen des Krieges und in den Mitteln, ihn zu beenden. Die Mehrzahl der Sozialisten betrachtet den Krieg als Ausfluß der gesellschaftlichen Ordnung. Ein Krieg läßt sich nicht vermeiden, ohne die Gesellschaft umzugestalten, das Eigentumsrecht abzuschaffen oder wenigstens einzuschränken. Einige Sozialisten sehen die Möglichkeit einer Sicherung des Friedens ohne radikale Umgestaltung der Gesellschaft. Considérant, Godin, Pecqueur, Vidal und Owen schlagen, sogar im Rahmen der liberalen Gesellschaft, eine europäische oder weltumfassende Föderation vor.

Alle sind sie jedoch davon überzeugt, daß die Einführung des Sozialismus den Krieg ausbannen wird. Ohne Eigentum, Konkurrenz, Ausbeutung und Korruption würde es gar keinen Grund zum Kriegführen geben. Dann bedarf es zur Sicherung des Friedens auch keiner übernationalen Organisation. ${ }^{1}$

Das allgemeine Interesse der Ökonomisten des neunzehnten Jahrhunderts für die Kriegsfrage erklärt sich aus der stark gewachsenen 1 E. Silberner, a.a.O., S. 220-235. 
Bedeutung des Wirtschaftsfaktors für den Verlauf der allgemeinen Geschichte. Sämtliche Richtungen, wie stark ihre Ideen auch auseinandergehen, anerkennen Zusammenhänge zwischen dem Frieden und den internationalen wirtschaftlichen Verhältnissen. Die Kriegsgeschichte gibt uns das Kriterium an die Hand, zu beurteilen, welche Auffassung damals die größte Gültigkeit besaß. Die unversöhnliche Rivalität zwischen Frankreich und England in allen vorangehenden Jahrhunderten, in denen die Staatspolitik über Krieg und Frieden entschieden hatte, kam nach I 860 endlich zur Ruhe. Zwischen diesen zwei Hauptmächten hat seitdem kein Krieg mehr gedroht, ausgenommen die kurze Spannung nach der Begegnung bei Faschoda.|Den meisten Autoren, zu welcher Richtung sie sich auch bekennen mögen, ist jedoch gemeinsam, daß sie das Kriegsproblem auf einen einzigen gesellschaftlichen Faktor reduziert haben. Keine einzige Richtung hat diese Erscheinung in ihrer Totalität verstanden, weil sich die Untersuchung auf den wirtschaftlichen Faktor beschränkte. Die wirtschaftliche Billigkeit hat nicht nur zwei Aspekte, sie hängt auch von anderen menschlichen Triebfedern ab.

Um die Jahrhundertwende gelangte die sozialistische Theorie vom Außenhandel zu einer gewissen Entwicklung. Im Jahre I894 stellte Jean Jaurès im französischen Abgeordnetenhaus einen Antrag zum Aufbau eines Staatsmonopols für den Getreidehandel. Die französischen Sozialisten waren an niedrigen Brotpreisen interessiert mit Rücksicht auf ihren Anhang in den Städten, aber zugleich wünschten sie, daß die Getreidepreise in solcher Weise beibehalten würden, daß sie dem französischen Bauern Gewinn einbringen würden. Frankreich hatte verhältnismäßig die größte agrarische Bevölkerung und der Sozialismus hatte dort auf dem Lande mehr Anhang gefunden als in anderen Ländern. Das Getreidemonopol sollte dazu verwendet werden, den einheimischen Produzenten höhere Preise zu zahlen als den ausländischen.

In Deutschland war es Rudolf Hilferding, der aus der Fülle der in Parteikreisen verfaßten einschlägigen Literatur eine neue sozialistische Theorie abzuleiten wußte. Im Gegensatz zu Marx vertrat er nicht die Ansicht, daß mit einem Sinken der Nahrungsmittelpreise ein entsprechender oder gar unproportionierter Abbau der Geldlöhne verbunden sein würde. Bei der bestehenden Macht der Industriegewerkschaften würde diese Folge nicht mehr einzutreten brauchen. Die Meinungen über die Interessen der Industriearbeiter waren geteilt. Manche Gewerbezweige hatten sich schon so weit entwickelt, daß sie ohne Protektion bestehen konnten, aber überall dort, wo dies nicht der Fall war, sahen die Arbeiter als Produzenten gern, daß ihnen 
Schutz gewährt würde. Sich eindeutig für den Freihandel erklären sei somit unmöglich.

Hilferding suchte eine Verantwortung zu finden für die Anpassung der Prinzipien an die sich widersprechenden praktischen Bedürfnisse. Hierzu unterschied er zwischen vier Arten von Tarifen, die sich im Wohlstandsniveau verschiedenartig auswirken: indifferente Tarife, progressive, reaktionäre Tarife und Kartelltarife. Bei den ersten drei Kategorien handelt es sich stets um Industrien, die ohne Schutzzölle nicht bestehen können. $\mathrm{Ob}$ ein Tarif indifferent, progressiv oder reaktionär ist, hängt nicht von seiner unmittelbaren Wirkung auf die Löhne ab, sondern von dem langfristigen Einfluß auf den allgemeinen Entwicklungsgrad des Wirtschaftslebens, d.h. auf die Anhäufung von Kapital, die als das Kennzeichen kapitalistischer Entwicklung gilt. Der Tarif ist indifferent, wenn er auf die Kapitalakkumulation keinen Einfluß hat; progressiv oder erziehend, wenn er die Arbeitsleistung im Produktionsprozeß steigert; reaktionär, wenn er verhindert, daß mehr Arbeit gebraucht wird, als es der Fall sein würde, wenn kein Tarif erhoben würde. Bei der vierten Kategorie besteht die Absicht, der Monopolbildung Vorschub zu leisten, wodurch der Preis im eigenen Lande über den Weltmarktpreis hinaussteigen kann und ein Monopolgewinn erzielt wird. Dies geschieht auf Kosten aller Verbraucherklassen. Wenn das Kartellprodukt als Produktionsmittel für Fertigfabrikate verwendet wird, verringert sich dadurch die Gewinnspanne der sich entwickelnden Industrien. Die Verkleinerung der Gewinnspanne in diesen Industrien beschleunigt den Prozeß der Kapitalanhäufung. ${ }^{1}$

Für die Zeit bis 1914 ist die sozialistische Analyse des Problems der Handelspolitik nahezu alles, was sich an normativer Kritik an dem herrschenden System erwähnen läßt. Aktiver Widerstand gegen die agrarische Protektion ist nicht daraus erwachsen. Die Werbung des Anhangs unter den Kleinbauern und den Landarbeitern wäre undenkbar gewesen, wenn die sozialistischen Parteien in den verschiedenen Ländern nicht die Reform des Grundbesitzes, die Verbesserung des landwirtschaftlichen Kredits und die agrarische Protektion, mochte diese auch reaktionärer Art sein, in ihr Programm aufgenommen hätten. Während des Krieges zwangen die Verhältnisse die Regierung, bei der Getreideversorgung zu intervenieren. Besonders in Zentraleuropa und in den skandinavischen Ländern hat man nach dem Krieg bei dieser Intervention ganz oder zum Teil beharrt. Die wirtschaftliche Argumentation verbirgt sich dann aber hinter politischen Motiven, am meisten in der Sowjetunion. Eine weitere Entwicklung dieser Theorie hat denn auch nicht stattgefunden.

1 B. F. Hoselitz, a.a.O., S. 235-237. 
Auch im Lager der Liberalen entwickelte sich die Theorie nur träge. Marshall und Taussig versuchten die Lehre von den komparativen Kosten der Wirklichkeit näherzubringen, indem sie die Annahmen, die einfachheitshalber als Gegebenheiten anerkannt worden waren, aus der Analyse eliminierten. ${ }^{1}$ Auf der monetären Seite war für eine Weiterentwicklung kaum Raum, weil die Technik des Zahlungsverkehrs keine Möglichkeit zu Manipulationen mit Wechselkursen bot.

Nach dem Krieg gerät alles in Bewegung. Zuerst auf monetärer Seite. In den Jahrgängen I91 8-1922 der Zeitschrift „De Economist” wird ein Federkrieg geführt über das Gleichgewicht der Zahlungsbilanz und die Erklärung der Wechselkurse. Zahlungsbilanztheorie gegen Kaufkraftparitätstheorie. ${ }^{2}$ Diese Polemik war die Reaktion auf die Entwicklung in der ausländischen Literatur. Auch der Lehrsatz von den komparativen Kosten wurde in zahlreichen Schriften erneut untersucht und auf seine Richtigkeit hin geprüft. Mit verschwindenden Ausnahmen, Ohlin und Taussig, haben alle Autoren das Prinzip aufrechterhalten, jedoch die Anwendbarkeit blieb mit der vielgestaltigen Wirklichkeit auf sehr gespanntem Fuß.

Das Ergebnis der Entwicklung sowohl der reinen Theorie als auch der monetären Theorie war, daß schon vor I939 die Theorie vom internationalen Handel den Vollständigkeits- und Geschlossenheitscharakter, den sie seit Stuart Mill beibehalten hatte, völlig verliert. ${ }^{3}$ Diese Tatsache steht nicht vereinzelt da, sondern sie ist ein Ausfluß entsprechender Veränderungen in der allgemeinen Wirtschaftslehre.

Das Streben nach einem Maximum an Reichtum hatte im Laufe der Zeit immer deutlicher gezeigt, daß es nur eine sehr mangelhafte Umschreibung des Zieles darstellt, das der Mensch bei seinem Streben nach Wohlstand im Auge hat. Bei der Kapitalanlage wählt der eine die höchste Verzinsung und übernimmt ein großes Risiko für die Hauptsumme, der andere zieht ein geringeres Risiko vor, nimmt dadurch einen niedrigeren Ertrag mit in Kauf. Die Alternative beeinflußt weitgehend die Verteilung der Investierungen, ganz besonders auch im internationalen Verkehr. Ähnlich liegen die Verhältnisse bei der unvollständigen Konkurrenz. Ein Monopolist, der seinen maximalen Monopolgewinn erzielt, hat nicht die Gewißheit, daß dies auf die Dauer am vorteilhaftesten ist. Es regt nicht nur die Konkurrenz

1 G. Stavenhagen, a.a.O., S. 41 2-414.

${ }^{2}$ F. J. de Jong, Phoenix Vrijhandel, Van het naĩeve naar het critische vrijhandelsstandpunt, De Economist, Bd. I00 (1952) S, 930, 942-947.

' G. Stavenhagen, a.a.O., S. 408 . 
an, sondern löst auch die staatliche Intervention aus, sei es im Inland oder im Ausland.

Die der alten Formel anhaftenden Mängel führten zu einer konsequenten Subjektivierung des wirtschaftlichen Ziels, das auf die Formel gebracht wurde, der Mensch strebe nach einer maximalen Befriedigung seiner Bedürfnisse. Die moderne Theorie geht von dem ganzen System sämtlicher vorhandenen Vorzugsurteile aus. Bereits die Historische Schule hatte die Forderung gestellt, die Ökonomie solle von dem Menschen, wie er wirklich ist, ausgehen. Die moderne Theorie ist aber der Historischen Schule in der weitergehenden Spezifikation der Motive nicht gefolgt. Die Theorie der Wahlhandlungen läßt den Inhalt der Triebfedern völlig dahingestellt. Sie geht von einigen Tatsachen aus: der Knappheit, dem Bestehen einer Rangordnung zwischen den Bedürfnissen und den alternativen Gebrauchsmöglichkeiten der Güter, die überall verwirklicht sind und somit einen völlig empirischen Charakter haben.

Mit dieser Theorie wurde das alte Ideal verwirklicht, eine Theorie, die nicht an Zeit und Ort gebunden ist und für alle Menschen gilt. Dieser erhöhte Wirklichkeitswert konnte nur auf Kosten einer Verarmung des Inhalts der Theorie erreicht werden. Bei den immer weniger eng formulierten Annahmen wurde sie auch immer ,inhaltsleerer", um schließlich zu einer völlig leeren Phrase herabzusinken. Über den tatsächlichen Verlauf des Handelns kann sie an sich keine Einsicht verschaffen, weil nämlich der Inhalt der Zielsetzungen und der Zweckmäßigkeitsgrad völlig unberücksichtigt bleiben.

Wenn es erlaubt wäre, die neue Lehre mit der Logik des Wählens zu identifizieren, so wäre der Preis für die Verbesserung gewiß zu hoch gewesen. In der rein formalen Umschreibung steht sie sogar hinter der klassischen Theorie zurück, in der, wenigstens bis zu einem gewissen Grade, der Verlauf des Handelns bestimmt war. Das leere Schema ist für die Behandlung wirtschaftlicher Probleme nur dann brauchbar, wenn man es mit den Angaben über das konkrete Handeln „ausfüllt". Mit dem Studium der differenzierten Motive hofft die allgemeine Ökonomie über den beschränkten Wirklichkeitswert der alten Schulen hinauszukommen. Die Zahl der Verhaltensweisen, die dafür in Betracht kommen, hat sich im Laufe der Jahre allmählich gemehrt. ${ }^{1}$

Die neue Methodik der allgemeinen Ökonomie hat zur Folge, daß im Begriff Rationalität zwischen der Zweckmäßigkeit und der Billigkeit ein anderes Verhältnis entstanden ist. Das zeigt sich am klarsten,

1 P. Hennipman, a.a.O., S. 397-434; F. J. de Jong, Over de betekenis van het begrip „rationeel handelen” in de economie, De Economist, Bd. 97 (1949), S. 48 1-484. 
wenn man der direkten Verantwortlichkeit des modernen Staates gegenüber der Wohlfahrt der eigenen Untertanen Rechnung trägt. Uberall ist von Staats wegen eine Rangordnung aufgestellt in der Verteilung der Hilfsquellen auf die Glieder der Gemeinschaft und auf die verschiedenen Bedürfnisse eines jeden einzelnen Gliedes. Das bedeutet, daß eine gesellschaftliche Präferenzskala gehandhabt wird, wobei man nach einer sogenannten "sozialen Rationalität" handelt. ${ }^{1}$ Die Autorität, mit der dieses Präferenzsystem auferlegt wird, legt den Gedanken nahe, daß diese Rationalität einen objektiven Charakter besitzen dürfte. Dies braucht jedoch nicht der Fall zu sein. Selbst wenn der Wohlfahrtsstaat zugleich ein demokratischer Staat ist, so ist der Gehalt der sozialen Rationalität nicht höher als das mehr oder minder zufällige Resultat, welches das Ringen der „pressure groups" zu realisieren erlaubt. Objektive Quantifizierung der gesellschaftlichen Wohlfahrt ist eine Aufgabe, der die Wirtschaftspolitik nicht gewachsen ist.

Im Rahmen der internationalen Verhältnisse ist die Rangordnung der Präferenzen ungleich verwickelter. Die Zielsetzungen sind nahezu allgemein anerkannt und angenommen. Vollbeschäftigung steht seit dreißig Jahren im Vordergrund des Interesses, aber man hat noch nicht spezifiziert, ob dabei nationale oder internationele Präferenz beachtet werden soll. Das Bewußtsein der Schicksalsverbundenheit ist allgemein genug verbreitet, daß das Maximum an Welteinkommen an zweiter Stelle genannt wird. Die Zielsetzung wird dynamisch aufgefaßt, so daß eine maximale Wachstumsrate, soweit sich diese mit humanen Bedingungen für die Produzenten verträgt, miteinbegriffen ist. Dann folgt der Wunsch nach einem maximalen nationalen Einkommen, gleichfalls dynamisch aufgefaßt. Jetzt wieder zurück zum internationalen Schauplatz: eine billige Verteilung des reellen Welteinkommens. Daran schließt sich die billige Verteilung des reellen Nationaleinkommens an. Als sechster und siebenter Punkt folgen die Grundsätze, nach denen die etwaigen Widersprüche zwischen den vorhergenannten Desideraten zu behandeln seien. Relative Stabilität von Einkommen für die Produzenten, und zwar dahin aufzufassen, daß im Produktionsmuster Anpassungen durchgeführt werden sollen, ohne in Übergangszeiten katastrophale Abweichungen in den Einkommen zu verursachen. Die Reihe schließt mit der pflichttreuen Erinnerung an die bisher schwächste Leistung der Wohlfahrtsökonomie: inflatorischer Anstieg der Preise soll vermieden werden. ${ }^{2}$

Zwecks dieser Versöhnung rivalisierender Zielsetzungen versucht

1 F. J. de Jong, a.a.O., S. $47^{8}$.

D. L. Munby, Christianity and Economic Problems, London, 1956, S. 216. 
die Wirtschaftswissenschaft Instrumente wirtschaftlicher Politik zu schmieden. Die Kommunikation und die Verarbeitung der Forschungsergebnisse stagniert infolge der riesigen Literaturmenge, die diesem Gegenstand gewidmet ist. Die Bibliographie von Ellis und Metzler über die Periode seit dem Verschwinden des geschlossenen Charakters der Lehre vom internationalen Handel umfaßt bei ihrem Erscheinen im Jahre 1949 über 1600 Veröffentlichungen. ${ }^{1}$ Diese kaum noch zu bewältigende Fülle ist zum Teil den sehr vielen Artikeln zuzuschreiben, in denen „Modellbau” getrieben wird. Meist sind es intellektuelle Übungen ohne großen praktischen Nutzen. Van Meerhaeghe schiebt vielen Autoren die Absicht unter, sie pflegten die Kunst um der Kunst willen und sie bekümmerten sich wenig um den Realitätswert ihrer Theorien. ${ }^{2}$ Neben dem wachsenden Zweifel an der Anwendbarkeit der Abstraktionen gibt es Verlust an Exaktheit der Begriffe. In einer internationalen Versammlung von Wirtschaftlern stellte einer von den Anwesenden fest, daß nicht weniger als neunundzwanzig verschiedene Bestimmungen des Begriffs Zahlungsbilanz verwendet wurden. ${ }^{3}$

Für die Rationalität als Billigkeit ist das Problem zentral geblieben, wie die Vorteile des internationalen Verkehrs auf die Länder verteilt werden. An zwei Beispielen läßt sich erläutern, weshalb von einer allgemeinen Erklärung nicht mehr die Rede sein kann.

Nach der Rechenkunst der komparativen Kosten führt der internationale Handel in den beteiligten Ländern zur Steigerung der Produktion solcher Güter, deren Produktionskosten verhältnismäßig günstig sind und zur Einschränkung der Produktion, wenn die Kosten ungünstig sind. Der Preis, d.h. das Einkommen des relativ knappen Produktionsfaktors sinkt, der des relativ überreichen Produktionsfaktors steigt. In Agrarländern steigt der Grundzins, in den Industriegebieten sinkt er. In übervölkerten Gebieten steigt das Lohneinkommen, in spärlich bevölkerten Gegenden sinkt es. In kapitalkräftigen Ländern steigt der Zins, in kapitalarmen sinkt er, dies alles im Vergleich mit dem Niveau vor Beginn des internationalen Verkehrs. Nach der Ansicht Ohlins kann absolute Nivellierung der Faktorpreise nur bei internationaler Beweglichkeit der Produktionsfaktoren zustande kommen. Samuelson und Lerner sind der Meinung, daß die Produktionsfunktionen der einzelnen Länder, selbst bei fehlender Mobilität der Produktionsfaktoren, auf die Dauer homogen würden. Wenn dies der Fall wäre, würde es keiner Theorie vom internatio-

1 Readings in the Theory of International Trade, London, 3. Auf. 1958, S. 555-625.

$2 \mathrm{M}$. van Meerhaeghe, Recente Bijdragen tot de theorie der Internationale Betrekkingen, Gent, 1959, S. 9 I.

- Van Meerhaeghe, a.a.O., S. 42. 
nalen Handel mehr bedürfen. ${ }^{1}$ Wirklichkeitwert hat nur die beschränktere Schlußfolgerung, daß vom internationalen Verkehr eine Tendenz zur Nivellierung der Einkommen und der Produktionsfunktionen der einzelnen Länder ausgeht. Relative Unentwickeltheit ist daher dem Wirtschaftsleben selber inhärent. Jede neue oder verstärkte wirtschaftliche Aktivität in einem bestimmten Gebiet löst gegenüber anderen, oft entlegenen Ländern „back-setting”-Effekte aus, ebensogut wie sie auch ,Streu-Effekte” hervorruft, besonders für die direkten Nachbarländer. Das hat jeweils sich verlagernde Niveauunterschiede zur Folge, die sich in der Regel durch automatisch auftretende Egalisationen nicht aufheben lassen. Zu diesem Zwecke sind zielgerichtete Interventionen erforderlich, sei es von Staats wegen oder vonseiten der Unternehmungen. ${ }^{2}$

Das zweite Beispiel. Wenn man davon ausgehen kann, daß relative Unentwickeltheit sich durch eine verhältnismäßig globale Anpassung korrigieren läßt, so verbleibt dennoch die Frage zur Beantwortung, wie sich der Verkehr zwischen Ländern mit großen Wohlstandsunterschieden auswirkt. Seit Beginn der modernen Technik bestand Ungewißheit, ob die Erschließung unterentwickelter Länder für die Industriestaaten Marktvergrößerung oder Marktverkleinerung zur Folge haben würde. Zwar war es deutlich, daß während der Zeit des Aufbaus auf eine verstärkte Absatzmöglichkeit für Investierungsgüter gerechnet werden konnte, aber es war noch ungewiß, ob nicht mit der Zeit der entgegengesetzte Effekt eintreten würde, daß die gesteigerte Produktivität die neuen Länder wirtschaftlich autark machen würde, so $\mathrm{da} ß$ sie aufhören würden, als Absatzmärkte zu fungieren. Smith bekämpfte diesen Gedanken. Die Steigerung der Produktion und der gesteigerte Wohlstand würden den Umfang des internationalen Handels vergrößern. Die Geschichte der Schweiz und Schwedens beweist, daß Aufnahme in den internationalen Verkehr, bei niedrigen Wohlstandsniveau im Anfang, nicht nur zur Anpassung an die Nachbarländer führen kann, sondern daß sogar ein Wohlstandsniveau erreicht werden kann, das höher liegt als in den Ländern, wo die Produktivitätsverbesserung zuerst entstanden war.

Trotzdem ist der Zweifel geblieben und er beherrscht sogar einen großen Teil der neueren Literatur. Eine extreme Auffassung ist diese, $\mathrm{da} ß$ die Industriestaaten immer reicher und die Entwicklungsländer immer ärmer werden; diese Auffassung wird bestritten mit dem Hinweis auf die Entwicklung in den Vereinigten Staaten, in Kanada, Australien und Neuseeland. Will man, wie ziemlich allgemein ange-

1 Staatslexicon, Bd. IV, S. 402.

" De Taak van Nederland ten aanzien van de Ontwikkelingslanden, uitg. Nederlandsche Maatschappij voor Nijverheid en Handel, Haarlem, I962, S. 23. 
nommen wird, gelten lassen, daß der internationale Verkehr günstig ist, so bleibt noch das Verteilungsproblem. Es gibt Pessimisten, die vermuten, daß das Tauschverhältnis, d.h. das Verhältnis zwischen den Indexziffern der Preise von Ein- und Ausfuhr, für die zurückgebliebenen Länder immer ungünstiger und die Disparität mit den entwickelten Ländern immer größer wird. Auf rein wirtschaftlichen Gründen will es uns nicht einleuchten, weshalb dies der Fall sein sollte. Es würde bedeuten, daß diese Entwicklung dem Schicksal der Staaten, die im neunzehnten Jahrhundert den „Streu-Effekten" des europäischen Wohlstandes ihren Fortschritt verdankten, diametral entgegengesetzt wäre. Die außerwirtschaftlichen Faktoren entziehen sich dem Zugriff der Wissenschaft ganz, dem der Wirtschaftspolitik weitgehend. Der wichtigste Faktor dürfte die Bevölkerungsfrage sein. Der Bevölkerungszuwachs kann zu langsam fortschreiten, aber auch zu schnell. Bei Übervölkerung ist eine Steigung des Einkommens pro Kopf nicht zu erwarten, gleichviel, ob das Tauschverhältnis unverändert bleibt oder verbessert wird. ${ }^{1}$

Mit diesen Beispielen ist die Sachlage skizziert. Van Meerhaeghe hat nach Besprechung einer großen Anzahl Veröffentlichungen das Fazit gezogen, daß es unmöglich ist, für irgendeinen Teil der Theorie oder der Wirtschaftspolitik zu einem endgültigen Urteil zu gelangen. Er nennt u.a. die Unkenntnis der Folgen des Handels für die Faktorpreise, das Tauschverhältnis, den Multiplikator, die Elastizität und die wirtschaftliche Integration. Je nach den Umständen hat eine bestimmte Wirtschaftspolitik völlig verschiedene Resultate. In einer Welt von Planwirtschaft, von Staatshandel, von essentiell willkürlichen und inflexibelen nationalen Preisstrukturen und kontrollierter Instabilität der Wechselkurse ist die klassische Theorie nicht anwendbar. Es ist möglich, daß für eine solche Welt eine relevante allgemeine Theorie überhaupt nicht bestehen kann. ${ }^{2}$ Im Zusammenhang mit dem Gerücht von der Bedeutung der europäischen Integration für die internationalen wirtschaftlichen Beziehungen ist der Schluß, zu dem L.H. Janssen in seiner vor kurzem erschienenen Dissertation gelangt, sehr ernüchternd. Die Benelux, die in ihren Anfängen am wenigsten protektionistisch war, wird von der EWG den größten Nutzen haben. Westdeutschland wird ungefähr dieselben Erträgnisse erzielen und Frankreich und Italien werden zusammen mit den Außenseitern einen kleinen Verlust zu verzeichnen haben. Nach einer Zeitspanne von zehn bis zwanzig Jahren werden die Veränderungen gänzlich zu vernachlässigen sein. An sich betrachtet ist die Zollunion kaum etwas Er-

1 Staatslexicon, Bd. IV, S. 403.

${ }^{2}$ Van Meerhaeghe, a.a.O., S. 92-93. 
strebenswertes, aber mag sie auch ein notwendiger Bestandteil einer wirklich fruchtbaren Zusammenarbeit sein, so werden weder die Außenseiter, noch Frankreich und Italien viel Gründe haben, ihre Folgen zu fürchten. ${ }^{1}$ Der Fall erinnert an die fleißige Jagd auf Kolonialbesitz im imperialistischen Zeitalter, dessen Bedeutung für den eigenen Wohlstand nicht wenig überschätzt wurde, obgleich am wenigsten von den Ökonomen. ${ }^{2}$

\section{IV}

Es gibt also eine doppelte Parallele zwischen der Entwicklung in der allgemeinen Wirtschaftslehre und der Theorie von den internationalen wirtschaftlichen Beziehungen. In der allgemeinen Wirtschaftslehre ist die Rationalität zur sozialen Rationalität evolviert. Im internationalen Verkehr ist die Verteilung der Vorteile auf die Staaten nicht länger eine selbstverständliche Folge der Rationalität, sondern ein Problem, und zwar ein Problem, dem normative Bedeutung zuerkannt wird. Die zweite Parallele ist, daß in beiden Fällen die Spannung zwischen Zweckmäßigkeit und Billigkeit durch Anlegen politischer und sozialer Maßstäbe gelöst werden muß.

Neben diesen Berührungspunkten besteht ein Unterschied. Das subjektive Element, das zum Begriff Rationalität hinzugekommen ist, führt zu auseinandergehenden Auffassungen. Innerhalb der nationalen Staaten gibt es die politische Autorität, welche die Präferenzen festsetzt. Im internationalen Verkehr hat eine wirksame Autoritätsinstanz immer gefehlt. Die Weltwirtschaft bis 1914 kennzeichnete sich gerade dadurch, daß sie nicht organisiert, geschweige denn institutionalisiert war. Eben diesem Umstand verdankte sie ihren Erfolg. Dieser Unterschied zwischen dem Bestehen einer Autorität für die Bestimmung der sozialen Rationalität und dem Fehlen einer entsprechenden Instanz für die Festsetzung der internationalen Präferenzen hat zu einem Widerspruch in der jüngsten Entwicklung der internationalen Beziehungen geführt. In der ersten Periode der Wirtschaftslenkung, etwa die Zeit zwischen 1920 und 1940, hat sich die politische Gewalt auf der ganzen Welt für den nationalen Wohlstand verantwortlich zu fühlen gelernt. Damit war eine Wirtschaftspolitik verbunden, die sich um die Neben-

I L. H. Janssen, Vrijhandel, Protectie en Tolunie, o.J., (1960) S. 148; vgl. C. Weststrate über den örtlich-indifferenten Charakter eines Teils der internationalen Spezialisierung: De Economist, Bd. 9o (194I) S. 34-60; und A. J. Brown über die abnehmende Komplementarität der internationalen Arbeitsteilung: A. J. Brown, Introduction to the World Economy, London, 2. Aufl. 1960, S. I45-163.

2 Henti Brunschwig, Mythes et Realités de l'Impérialisme colonial français 1870-r914, Paris, 1960, S. 23-30. 
wirkungen der Sorge um die eigene Wohlfahrt in der internationalen Gesellschaft nicht kümmerte. Nach I945 hat man keinen Rückfall in dieses System, das sich als verhängnisvoll erwiesen hatte, gewollt. Also kein internationaler Verkehr auf der Grundlage einer autonomen Wirtschaftspolitik. Andererseits aber hat der heutige Staat für die Nationalwirtschaft eine nahezu totale Verantwortung übernommen und stehen ihm weit wirksamere Hilfsmittel zur Verfügung, um die nationale Wohlfahrt nach seinen Einsichten zu beeinflussen. Er wird nicht zulassen, daß externe Faktoren der internen sozialen Rationalität irgendwie schaden.

Welches ist die Resultante dieser rivalisierenden Kräfte? Praktisch gesprochen, daß es bei weitem nicht eine ausgemachte Sache ist, ob sich dieser Nationalismus, noch verstärkt durch den Regionalismus, auf die Dauer mit den strikten Voraussetzungen für einen ordentlich funktionierenden Weltverkehr vertragen wird. Was die Theorie betrifft, sie hat nicht nur ihre Allgemeinheit und Geschlossenheit verloren, sondern sie ist auf dem Wege, ein Bestandteil zu werden von etwas, das als die Theorie oder die Lehre von der internationalen Gesellschaft bezeichnet werden müßte. Sie begreift außer dem Wirtschaftsleben die Staats- und Militärpolitik, die Soziologie, die Demographie, kurz das ganze kulturelle Leben ein. Eine echte Theorie ist sie somit nicht. Ein amerikanisches Buch, das sich diesem Thema widmet, erschien unter dem Titel „Theoretical Aspects of International Relations".1 Generalisierende Schlüsse aus feststehenden Daten finden sich darin nicht, es sei denn in einer formalisierten Gestalt, genauso wie es bei der allgemeinen Ökonomie der Fall ist.

Einer von diesen Schlüssen berührt sehr deutlich die Ungewißheit der Stellung, die der nationale Staat, sogar der größte, in dem heutigen Weltsystem einnimmt. Er strebt nach sozialer Rationalität auf eigenem Boden und, sehr tugendhaft, nach Koordination dieses Ziels mit dem Wohl anderer Völker. Die Schwierigkeit, diese Rivalität in den Zielsetzungen zu überbrücken, ist nicht so sehr, daß die eigene Wohlfahrt nur auf Kosten der Wohlfahrt anderer gedeihen kann, als vielmehr, daß man nicht weiß, welches die Folgen des Termingeschäfts mit dem Ausland sind für die physischen, intellektuellen und materiellen Hilfsquellen, die alle zusammen die nationale Volkskraft bestimmen; ebensowenig, wie sich verhältnismäßig die Volkskraft der Nachbarstaaten, der Bundesgenossen und der anderen Rassen und ideologischen Gegner entwickeln wird. Der nationale Staat ist sich dieser seiner größten Schwierigkeit bewußt und diese bestimmt sein

- Theoretical Aspects of International Relations, ed. William T. R. Fox, Indiana, r959; vgl. R. Bosc, La société Internationale et l'Eglise, Sociologie et Morale des Relations Internationales, Paris, $196 \mathrm{I}$, S. $315-328$. 
ganzes Handeln. „In the long run the national interest is the preservation of the community". Wohlverstanden, die nationale Gemeinschaft. Welchen Raum gibt es dann noch, nicht in den primitivsten Kampf aller gegen alle zu verfallen? Hierüber liegen keine Lehrsätze vor, die einigermaßen allgemein anwendbar wären. „The short-run national interest is more difficult to identify. Frequently the means are confused with the end". ${ }^{1}$ Die Staats-, Militär- und Wirtschaftspolitik von einem Tag zum anderen und von einem Jahr zum anderen beruhen noch ebensosehr auf Improvisation und Opportunismus wie in den Zeiten des sog. politischen Gleichgewichts.

Unter solcher Betrachtung läßt sich ein Rückgang feststellen im Vergleich zum Kosmopolitismus von vor hundert Jahren, wieviele Mängel ihm auch angehaftet haben mögen. Rationalität war identisch mit heilsam und billig, wenigstens im internationalen Verkehr. Dieses Vertrauen zu der Parallelität von nationalem und internationalem Wohlsein auf lange Sicht dürfte die einzige Erklärung dafür sein, daß eine einigermaßen fundamentale normative Kritik an der Konzeption so lange hat fehlen können. Hilferdings Theorie hatte eine beschränkte Tendenz und blieb außerdem eine Theorie. Die Politik der deutschen Sozialisten blieb auf den Freihandel ausgerichtet, ebenso wie die der englischen, französischen und italienischen Sozialisten. ${ }^{2}$ Die Programme der übrigen politischen Parteien in Deutschland sahen darin auch kein Problem. ${ }^{3}$ Das russische kommunistische Parteiprogramm des Jahres 1903 nimmt als Ausgangspunkt, daß die gegenseitige Abhängigkeit der kapitalistischen Länder immer größer geworden ist, richtet aber seine Kritik auf die gesellschaftliche Ordnung im allgemeinen. ${ }^{4}$ Auch in den sozialen Enzykliken bleibt das Problem unberücksichtigt. Rerum Novarum erwähnt es mit keinem Wort, dasselbe gilt für alle folgenden Enzykliken Leos XIII., Pius' X., Benedikts XV., und Pius' XI., wie auch für die sozialen Briefe der Episkopate in Europa und Amerika, die zwischen I891 und I931 in einer Anzahl von über 1700 erschienen sind. ${ }^{5}$

In der Enzyklika Quadragesimo Anno des Jahres 193 I fand sich zum erstenmal ein Passus, der die Aufmerksamkeit ausdrücklich auf die internationalen wirtschaftlichen Beziehungen hinlenkte. Die Schick-

1 Theoretical Aspects of International Relations, S. 69-8 2.

2 B. F. Hoselitz, a.a.O., S. 233.

8 Wilhelm Mommsen, Deutsche Parteiprogramme, München, 1960, S. 380; Wolfgang Treue, Deutsche Parteiprogramme 1861-1956, Göttingen 1956, S. 17,22.

- Boris Meissner, Das Parteiprogramm der Kommunistischen Partei der Sowjet-Union I903 bis I961, Köln, I962, S. IIs.

- La Hiérarchie catholique et le Problème social depuis l'encyclique „Rerum Novarum” I 891-193 I, Répertoire bibliographique des documents émanés des Souverains Pontifes et de l'Episcopat, [Paris] 1931. 
salsverbundenheit der Nationen in der wirtschaftlichen Ordnung sollte ein Anreiz zur Zusammenarbeit sein. Durch Vereinbarungen und Institutionen sollten sie nach einer glücklichen internationalen wirtschaftlichen Interessengemeinschaft streben. ${ }^{1}$ In den Niederlanden stellte der katholische Arbeiterverband 1938 ein Programm auf, in dem er seine Mitwirkung an der Gründung einer internationalen Ordnung zusagte, die auf den Prinzipien des Rechts beruhen sollte. ${ }^{2}$ Wem diese Mitwirkung hätte geleistet werden müssen, wird damals wohl eine offene Frage geblieben sein.

Pius XII. drang zum Kern des Problems durch in seiner Ansprache an den Congrès des Échanges Internationaux am 7. März 1948. Die Nationalwirtschaft bilde eine natürliche Einheit. Sie dürfe eine möglichst vielseitige Entfaltung all ihrer Produktionsmöglichkeiten auf dem ganzen Gebiet, über das sie sich erstrecke, beanspruchen. Demzufolge hätten die internationalen wirtschaftlichen Beziehungen durchaus eine notwendige Funktion, wenn auch nur eine sekundäre. „Die Umkehrung dieses Verhältnisses ist einer der größten Irrtümer der Vergangenheit gewesen". ${ }^{3}$

Die Prinzipien des Rechts, auf die der Arbeiterverband hinzielte, und die Rangordnung, die Pius XII. angegeben hat, sind zentrale Fragen der internationalen Ordnung. Es ist nicht wahrscheinlich, daß der Arbeiterverband von empfindsamem Heimweh nach der Rechtsordnung des neunzehnten Jahrhunderts erfüllt war. Dennoch war es die Zeit, welche den Rechtsstaat geschaffen hat und diese Errungenschaft hatte sich für das internationale Recht als ebenso unentbehrlich erwiesen wie für die nationalen Gesellschaften. In der inneren Politik war das Wirtschaftsrecht im Laufe der Zeit differenziert und verfeinert worden, aber auch relativiert. Das Rechtsbewußtsein hat mit dieser Evolution nicht Schritt zu halten vermocht. Allein schon die ungeahnten Schwankungen im Geldwert haben den Zusammenhang zwischen Leistung und Belohnung, zwischen der Kapitalbildung und den Erträgnissen zerrissen. Es zeigt sich, daß die Rechtsverfeinerung auf die Dauer hart an Willkür und Ungerechtigkeit streift; davon gibt die Dekolonisation die empörendsten Beispiele. Wenn der Rechtsstaat

1 J. Y. Calvez et J. Perrin, Eglise et Société économique, l'Enseignement social des Papes de Léon XIII à Pie XII 1 878-1958, Paris, 1959, S. 391 .

2 Honderdvijftig jaar Katholieke Arbeidersbeweging in West-Europa 1789 -1939, Brüssel, 1961, red. S. H. Scholl, Brüssel, 1961, S. 352 ; Charles Flory, Le Catholicisme social devant la crise de la Civilisation, s.l., 1960, S. 89-106; Het Plan van de Arbeid, rapport van de commissie uit N.V.V. en S.D.A.P., Amsterdam, 1935, S. 21 : „Het Plan van de Arbeid” ist freilich national, aber er kann direkt in einen internationalen Rahmen gestellt werden. Das Evangelische Soziallexikon, ed. Friedrich Karrenberg, Stuttgart, 3. Aufl., 1958, verschafft keine Information über den evangelisch-christlichen Standpunkt. 3 La Documentation catholique, t. 54, Paris, 1948, col. 623-626; J. Y. Calvez, a.a.O., S.393. 
noch erfunden werden müßte, so würde das eine schwierigere Aufgabe sein als vor einem Jahrhundert. Trotzdem ist dies für den internationalen Verkehr das Hauptanliegen dieser Zeit.

Der Ausspruch des Papstes Pius XII. kann nicht bedeuten, daß er die Rückkehr zu dem autonomen Primat der nationalen Staaten hätte befürworten wollen, wie dieses Prinzip in der Zeit zwischen den beiden Weltkriegen angewandt wurde. Was er denn wohl bedeutet, kann man nur dann in Erfahrung bringen, wenn man die beiden Komponenten der Rationalität weiterhin sorgfältig untersucht. Dieser Prozeß läßt sich nirgends deutlicher verfolgen als im christlichen sozialen Denken in Frankreich während der letzten Jahrhunderthälfte. ${ }^{1}$

Man unterscheidet drei Richtungen. Die erste Gruppe legt den Akzent hauptsächlich auf die Regeln der Billigkeit. Thre Autoren sind antikapitalistisch, antikommunistisch und auch antidirigistisch. Sie befürworten eine „Bedürfniswirtschaft”. Sie verlangen, daß ein Prioritätsschema von Bedürfnissen aufgestellt werde. Die dringendsten Bedürfnisse sollen den Vorzug vor allen anderen bekommen. Luxus ist nicht erlaubt, solange in der Welt noch Hunger gelitten wird.

Mit diesen absoluten Prinzipien ist die wirtschaftliche Seite der Rationalität auf das Mimimum zurückgeführt. Wenn man dies gewollt und seit Anfang der Zeiten folgerichtig durchgeführt hätte, so wäre nie eine Kathedrale erbaut und nie ein Buch geschrieben worden. Wie die Preisbildung verläuft und wie die Verteilung der Produktionsfaktoren auf die alternativen Anwendungsmöglichkeiten geregelt wird, bleibt im ungewissen.

Die zweite Gruppe ist der Ansicht, es sei notwendig, zwischen Marktwirtschaft und Planwirtschaft zu wählen, und sie wählt letztere. Mit den Kommunisten hat sie den Glauben an einen Heilstaat gemeinsam. Der Kollektivität kommen Vorrechte zu vor den niedrigeren Gemeinschaften und vor dem persönlichen Recht. Teilhard de Chardin bezeichnet dies als „planétisation”. Es wird eine Zeit anbrechen, da die Menschheit nicht mehr sein wird als ein einziges Wesen, die Individuen nicht mehr sein werden als bloße Zellen. Das Einzelwesen wird sich damit abfinden müssen, zu verschwinden; es wird ihm nur übrig bleiben, an seine Kinder und an seine Werke zu denken, die es überleben werden.

Bei dieser Richtung finden wir also eine noch stärkere Neigung zum Rigorismus. Die erste Gruppe bedroht nur die wirtschaftliche Zweck-

1 Influence des expériences communistes sur les doctrines, Centre d'étude des pays de l'Est, Institut de Sociologie Solvay, Bruxelles, 1958, Daniel Villey, L'influence des expériences communistes sur la pensée économique et sociale d'inspiration chrétienne, S. $129-176$. 
mäßigkeit, die zweite führt die Überspanntheit der normativen Humanität ad absurdum.

Die dritte Gruppe will den Liberalismus in christlicher Gestalt „repenser”. Sie anerkennt weiterhin die Konkurrenz als die bewegende Kraft wirtschaftlichen Fortschritts. Die Risiken der Investierungen können vom Staat oder von den Unternehmern getragen werden. Von der Tatsache abgesehen, ob jener dies besser tut als diese wird die Entfaltung der schöpferischen Persönlichkeit in Nachteil geraten, wenn Erzeugung und Konsum dem Staat unterstellt werden. Aus diesem Grunde sprechen sie sich aus für eine freie, wenn auch sozial normierte Gesellschaft und für wirtschaftlichen Internationalismus als Konsequenz der freien Gesellschaft. Die Möglichkeiten zur Gerechtigkeit bestimmt letzten Endes die Transzendenz. Gott ist vom Geschaffenen getrennt. Dadurch ist in dieser Welt alles relativ, alles „mutuellement rival”, gegenseitig entgegengesetzt. Es ist natürlich, $\mathrm{da} ß$ es ein Interessenspiel gibt, in dem die verschiedenen von den Menschen gesteckten Ziele miteinander wetteifern. In diesem Sinne ist die Welt in sich geteilt. Wahrheiten werden stückweise entdeckt und Ziele in der Zeit verwirklicht, also stufenweise. Der Tauschhandel ist das Gebiet unserer Materialität im Wetteifer schlechthin, sowohl in den unteren als in den oberen Gruppierungen. Es zeigt sich, daß der wirtschaftliche Internationalismus, auf einer ganz anderen Ebene als Bertil Ohlin meinte, nur einen Sonderfall des Tauschverkehrs im allgemeinen und der ihn beherrschenden Rationalität darstellt. 